\title{
Thermodynamic pre-determination of power generation potential in geothermal low-temperature applications
}

Henrik Öhman ${ }^{1 *}$ and Per Lundqvist ${ }^{2}$

\author{
* Correspondence: henrik@hohman.se \\ 1 Department of Energy Technology, \\ Royal Institute of Technology KTH \\ Brinellv, 68 Stockholm 10044, \\ Sweden \\ Full list of author information is \\ available at the end of the article
}

\begin{abstract}
Background: Small capacity, low temperature, geothermal heat sources provide significant opportunities for distributed, small scale power generation. Project definitions and pre-dimensioning however require advanced thermodynamic engineering at a cost independent of project size. In order to reduce this cost, a new method has been developed to allow basic level engineers to perform pre-optimisation of thermodynamic potential as well as expected performance using available power plant technology at such pre-optimized conditions.

Results: By reducing the complexity of second-law computations, a simple two-dimensional diagram is shown representing the dimensioning criteria required for maximum power generation using the particular heat source and sink while considering expected power plant performance, using systems and components on the market.
\end{abstract}

Methods: By sensitivity analysis the combination of thermodynamic analysis and real world data correlations was simplified and arranged for pre-dimensioning of business cases.

Conclusions: Optimal pre-dimensioning of power generation system for any geothermal heat source, with a defined heat sink, can be determined without advanced thermodynamic expertise. This reduces the cost for business case proposals, pre-dimensioning and tender specifications of small-scale power generation systems for low temperature heat sources. The intended implication of this work is to increase the use of low-temperature geothermal wells for distributed power generation.

Keywords: ORC; Kalina; Waste heat; Power cycle; Geothermal; Performance; Power; Model

\section{Background}

Well over 1,000 scientific works are published yearly on findings from research on Low Temperature Power Cycles (LTPC). Advanced cycle configurations are thoroughly investigated (Ho et. al 2012), as well as transcritical solutions (Vèles et. al 2012). Internal fluid selections for very specific geothermal applications (Bu et. al 2013) as well as general use of zeotropic mixtures (Heberle et. al 2012; Aghahosseini and Dincer 2013) constantly improve our knowledge. Yet, dissemination of the knowledge is slow and few scientific articles are published on results from real, operating field units. Technical developments of LTPCs benefit greatly from the findings available in

\section{Springer}


research. Site developers of geothermal wells however require references to real systems. The few reports of real, operating system performances therefore need to be carefully assessed and generalized in order to provide guidance to what a practitioner could achieve with a particular geothermal well.

Conventional second law analysis of power generation potential in low-temperature applications is vital, as well as a non-biased approach, to understand any particular case of LTPC application. As long as power plants of sufficient sizes are considered, cost of expert engineering is not prohibitive to projects. This workload is however very much similar regardless if a 50- or 0.5-MWe plant is considered. Obviously, the relative cost will then rise significantly for small-scale power plants and could prohibit the use of such.

In order to simplify and increase the confidence of second law analysis output, Öhman and Lundqvist (2012) suggested a simplified analysis method using a finite Carnot cycle analogy determining the Integrated Local Carnot Efficiency, $\eta_{c, l}$, as the potential ratio of output work, $\dot{W}_{\mathrm{NPO}}$, to input heat, $\dot{Q}_{1}{ }^{\text {a }}$.

$$
\eta_{c, l l}=\frac{1}{n} \sum_{i=1}^{n} \eta_{c, l}(i)
$$

where $i$ is a very small, finite Carnot process in a series of $n$ and $\eta_{c, l}$ is the Carnot efficiency of $i$. Note that the small, finite and constant size step is taken in the dimension of heat flux from the heat source and that 'finiteness' or apparent specific heat capacity of both source and sink is required to determine $\eta_{c, l}$. The reason for using this summation is, as described in (Öhman and Lundqvist 2012), the lack of possibility to solve the equivalent integral analytically. One may bear in mind that Integrated Local Carnot Efficiency is possible to derive by exergy analysis or entropy analysis, though an iterative procedure is required.

The function of $\eta_{c, l l}\left(\dot{Q}_{1}\right)$ is determined by ending the summation in Equation 1 at $\dot{Q}_{1}$. This also gives a relation of $\eta_{c, I l}\left(\psi_{U}\right)$, where $\psi_{U}$ is the utilization of the available heat transport from source to sink by a reversible power cycle.

Though the calculations of Integrated Local Carnot Efficiency is relatively simple and straight-forward (see Equation 1), it would still require the local engineer to set up a model in a suitable software by himself or purchase a commercial software, in order to perform his investigation. Making software is prone to cause errors and purchasing commercial software is likely to be non-attractive from a cost perspective.

Small-scale power plants, using low-temperature heat, LTPCs, have entered the market in a number of formats. Öhman and Lundqvist (2013) investigated a large sample of operating plants as well as commercially available, standardized LTPCs. It showed that performance of all plants is a function of the ratio of utilized to potential heat transport from heat source to heat sink.

With this knowledge, the need to simulate a particular thermal process, such as Organic Rankine cycles (ORC; Schuster et al. 2009), Kalina cycle (Walraven et al. 2013), Trilateral Flash cycle (TFC; Fischer 2011) or any other cycle becomes unnecessary for pre-optimizing plants and business cases.

In order to offer a correct and cost-efficient tool, a graphic model was therefore developed in order to allow the simplest possible access to Equation 1. Combined with a calculated absolute maximum rate of heat exchange from the heat source $\dot{Q}_{\mathrm{CA}}$, any 
practitioner is able to present a scientific best estimate of the maximum expected power output and the corresponding required utilization of available heat exchange between source and sink. This allows for estimating size and cost of the major cost items as well as the expected sales volume of electric power.

The authors' intention is to enhance the use of small-scale LTPCs by offering this simplified analysis procedure.

\section{Methods}

The Integrated Local Carnot Efficiency model is dependent on a number of parameters making a detailed graphic version multidimensional and too complicated. Therefore, a reduction of dependent parameters was performed by analysing the model sensitivity to each parameter.

Intuitively, we understand that the entry temperatures of source and sink, $T_{1}$ and $T_{2}$ and the thermal capacity of the fluid streams are parameters affecting the potential power generation of a power cycle. More often overlooked is the dependence on the utilization as defined in Equation 2.

$$
\psi_{U}=\dot{Q}_{1} / \dot{Q}_{\mathrm{CA}}
$$

where $\dot{Q}_{\mathrm{CA}}$ corresponds to the heat transfer rate from the source when exit temperature of source and sink coincide and work is produced entirely reversibly, see Equations 4 and 5. $\dot{Q}_{1}$ represents the particular rate of heat transferred from the heat source to the process.

Figure 1 shows the thermal entities of a general power cycle in an application with relatively low temperature difference between source and sink. As heat transfer from the source is used as the abscissa; the temperature gradient of the source flow is shown linearly. Please note that the graphic model developed in this article is ONLY valid for source/sink-streams with constant apparent heat capacity. If ice slurries, condensing

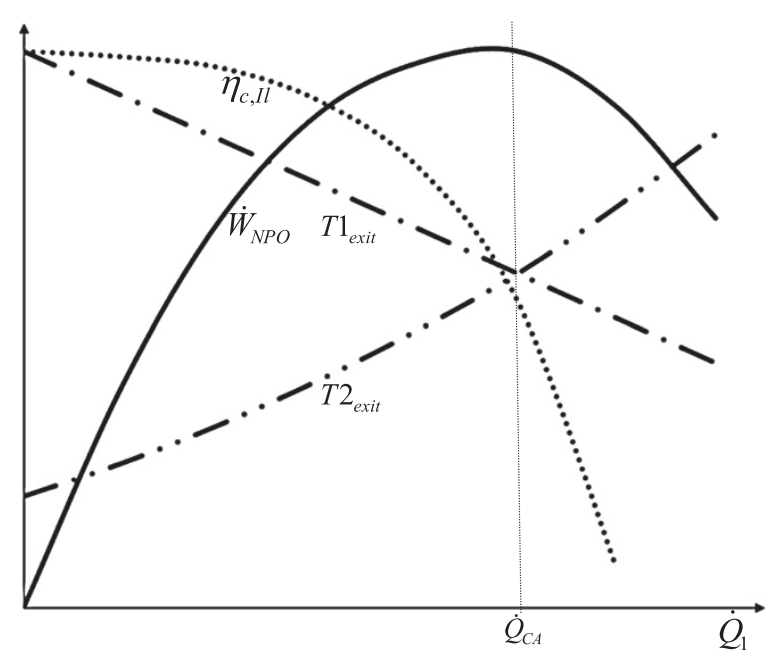

Figure 1 Logic representation of thermal entities. Exit temperatures, rate of work and Integrated Local Carnot Efficiency vs. rate of heat extracted from the heat source in a power cycle process, transferring heat between a finite heat source with entry temperature $T_{1}$ and a finite heat sink with entry temperature $T_{2}$. 
steam or other non-constant heat capacity streams are used; Equation 1 has to be calculated accordingly.

The Integrated Local Carnot Efficiency is also included in Figure 1. Obviously, it approaches the Carnot efficiency limit at a very low heat flux, $\dot{Q}_{1}$, from the source, to the left of Figure 2. As heat transfer rate from the source is increased, $\eta_{c, l l}$ diminishes and at $\psi_{U}=1, \eta_{c, I l}$ is identical to the Curzon-Ahlborn efficiency, as in Equation 3.

$$
\eta_{C A}=1-\sqrt{T_{2} / T_{1}}
$$

If $\psi_{U}$ is further increased, the power cycle has to perform heat pump duty and $\eta_{c, l l}$ will eventually become negative.

Maximum rate of heat transfer from the source in a reversible power cycle, operating between a defined source and sink is defined in Equation 4:

$$
\dot{Q}_{C A}=\left(T_{1}-T_{C A}\right) / \alpha_{1}
$$

Where the common exit temperature of source and sink is defined by Equation 5, according to Öhman and Lundqvist (2013),

$$
T_{\mathrm{CA}}=T_{1} \cdot \frac{T_{\mathrm{rat}}+\sqrt{T_{\mathrm{rat}}} \cdot \alpha_{\mathrm{rat}}}{1+\sqrt{T_{\mathrm{rat}}} \cdot \alpha_{\mathrm{rat}}}
$$

As explained in detail in the reference, Equation 5 is derived from the theoretical assumption of equalising the source and sink temperature by using a reversible power cycle to transport the heat. As the Curzon-Ahlborn efficiency is known at $T_{\mathrm{CA}}$, the latter can be expressed analytically.

The entry temperatures of source and sink are grouped to a temperature ratio as in Equation 6.

$$
T_{\text {rat }}=T_{2} / T_{1}
$$

The investigation of operating LTPC performance in (Öhman and Lundqvist 2013) resulted in a general, empirical correlation of the efficiency of real power cycles and

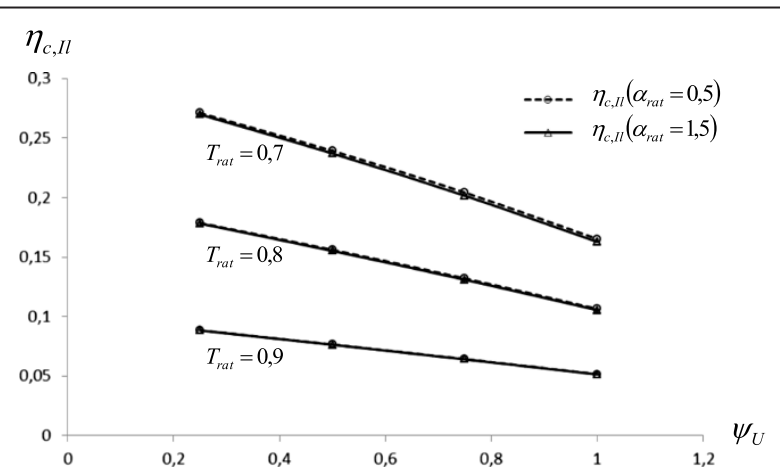

Figure 2 Integrated carnot efficiency calculated with $a_{\text {rat }}$ of $\mathbf{0 . 5}$ and $\mathbf{1 . 5}$. The sensitivity to $a_{\text {rat }}$ is low within the studied domain. 
utilization. That correlation is the key element in enabling a real power output estimation model and is shown in Equation 7

$$
\mathrm{FoC}=0.672 \cdot e^{-0.874 \cdot \psi_{u}}
$$

where fraction of Carnot, FoC, is defined as in Equation 8

$$
\mathrm{FoC}=\eta_{t h} / \eta_{c, l l}
$$

Note that Equation 7 is an empirical correlation based on a large amount of data.

\section{Temperatures}

Since Equation 1 suggests some degree of numerical error, a computational investigation was performed. In varying $T_{1}$ from 85 to $1,000^{\circ} \mathrm{C}$ with $\alpha_{\text {rat }}$ ranging from 0.5 to 1.5 , a total sensitivity of $10^{-5}$ was discovered. Thus, the absolute level of temperature was determined as irrelevant for the domain studied. Temperature ratios, according to Equation 6, were investigated separately.

\section{Heat source and heat sink streams}

The inverse apparent heat capacity of source stream is defined as Equation 9, as of (Öhman and Lundqvist 2012)

$$
\alpha_{1}=\Delta T_{\text {source }} / \dot{Q}_{1}
$$

and similar for the heat sink.

The inverse apparent heat capacities of the streams are then grouped to a ratio, as in Equation 10

$$
\alpha_{\text {rat }}=\alpha_{2} / \alpha_{1}
$$

A computational analysis was performed showing zero dependency of absolute level of $\alpha_{1}$ in Equation 1 using constant $\alpha_{\text {rat }}$ and variation in $\alpha_{1}$ of 1 to 10 . As long as $\alpha_{\text {rat }}$ is kept constant the absolute level of $\alpha_{1}$ is therefore considered as irrelevant to Equation 1.

The absolute level was therefore considered as irrelevant to the results of Equation 1.

Note that this is only true for combinations of source and sink with constant apparent heat capacities.

In order to further reduce the number of parameters, $\alpha_{\text {rat }}$ was investigated by a similar computational analysis. The result can be seen in Figure 2, showing a negligible sensitivity of $\alpha_{\text {rat }}$ at $T_{\text {rat }}=0.7$ while at 0.8 and 0.9 , no significant difference at all was observed.

From this, Equation 1 could be considered as independent of $\alpha_{\text {rat }}$ within the studied domain of $T_{\text {rat }}>0.7$ Note that this is the interesting domain for applications using lowtemperature heat sources. For solar heated power cycles and combustion heated power cycles, Equation 1 has to be calculated numerically due to the lower levels of $T_{\text {rat. }}$.

\section{Power estimation}

Net power output from a power cycle can be defined as in Equation 11. 


$$
\dot{W}_{\mathrm{NPO}}=\dot{Q}_{1} \cdot \eta_{\mathrm{th}}
$$

From Equations 2, 7 and 8, this can be written as Equation 12.

$$
\dot{W}_{\mathrm{NPO}}=\dot{Q}_{\mathrm{CA}} \cdot \Omega_{\mathrm{spec}, a}\left(\psi_{U}\right)
$$

where the last term, application-specific work ratio, is a variable grouping all dimensionless terms as of Equation 13.

$$
\Omega_{\text {spec }, a}\left(\psi_{U}\right)=\psi_{U} \cdot \operatorname{FoC}\left(\psi_{U}\right) \cdot \eta_{c, I l}\left(\psi_{U}\right)
$$

The reason for introducing $\Omega_{\mathrm{spec}, a}\left(\psi_{U}\right)$ is to create a dimensionless entity suitable for a two-dimensional calculation diagram as in Figure 3.

The user has to calculate $\dot{Q}_{C A}$ numerically according to Equation 4, based on his local application data. Then to calculate the expected net power output, $\dot{W}_{\mathrm{NPO}}$, using real equipment the user only need to identify the utilization in Figure 3, giving maximum $\Omega_{\text {spec, }, \text { }}$, and from there use Equation 12 to get the answer.

\section{Examples of calculation}

A sample geothermal application is defined according to Table 1 . Brine $\left(300 \mathrm{~m}^{3} / \mathrm{h}\right)$ is available as heat source and a local river can provide cooling water. The suitable need for cooling water pump capacity is unknown.

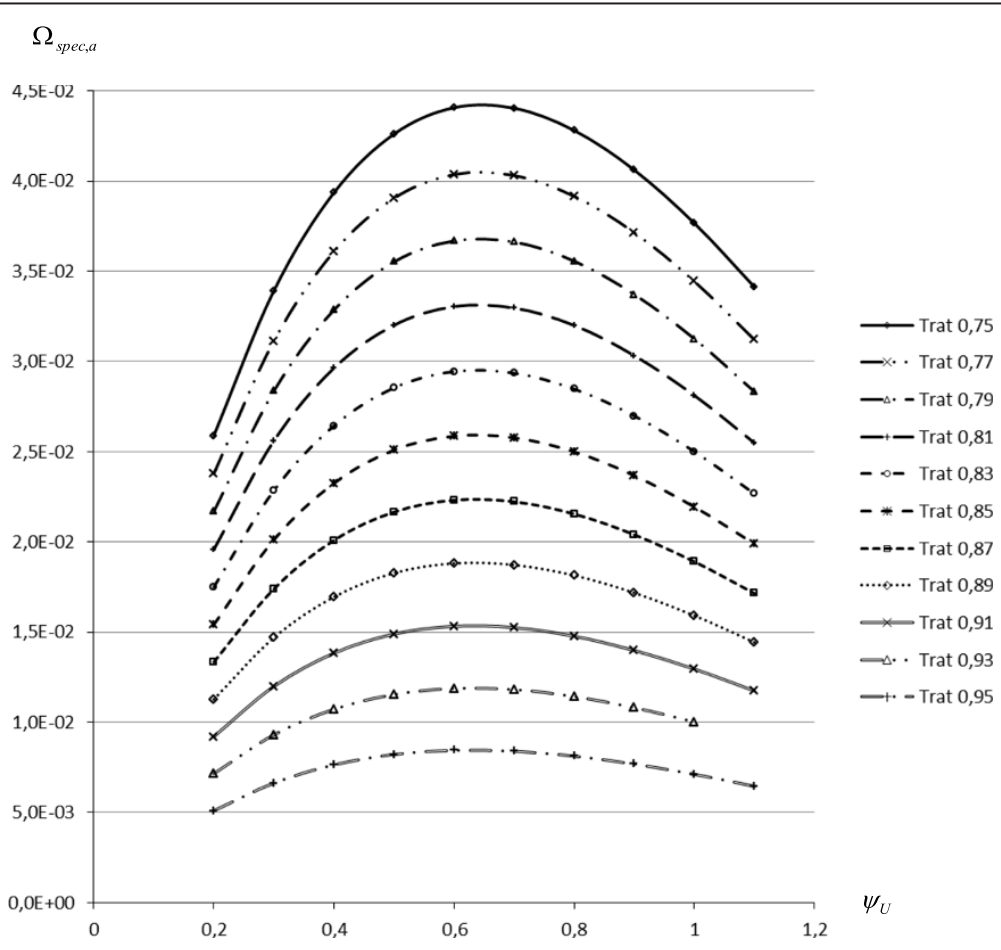

Figure 3 Application-specific work ratio. The ratio of real expected power output, using market products, to the theoretical heat transport from the heat source required to reach equal exit temperatures of source and sink while using a reversible power cycle. 
Table 1 Stepwise calculation results

\begin{tabular}{|c|c|c|c|c|c|c|c|c|}
\hline \multirow[b]{2}{*}{ Brine temp } & \multicolumn{3}{|c|}{ Winter } & \multicolumn{3}{|c|}{ Summer } & \multirow{2}{*}{$\begin{array}{l}\text { Unit } \\
{ }^{\circ} \mathrm{C}\end{array}$} & \multirow{2}{*}{$\begin{array}{l}\text { Source } \\
\text { Known }\end{array}$} \\
\hline & 105 & 105 & 105 & 105 & 105 & 105 & & \\
\hline Brine flow & 300 & 300 & 300 & 300 & 300 & 300 & $\mathrm{~m}^{3} / \mathrm{h}$ & Known \\
\hline River water & 10 & 10 & 10 & 25 & 25 & 25 & ${ }^{\circ} \mathrm{C}$ & Known \\
\hline Water flow & 150 & 300 & 450 & 150 & 300 & 450 & $\mathrm{~m}^{3} / \mathrm{h}$ & Assumed \\
\hline$a_{1}$ & 2.87 & 2.87 & 2.87 & 2.87 & 2.87 & 2.87 & K/MW & Equation 9 \\
\hline$a_{2}$ & 5.74 & 2.87 & 1.91 & 5.74 & 2.87 & 1.91 & K/MW & Equation 9 \\
\hline$T_{\text {rat }}$ & 0.75 & 0.75 & 0.75 & 0.79 & 0.79 & 0.79 & $(-)$ & Equation 6 \\
\hline$a_{\text {rat }}$ & 2 & 1 & 0.67 & 2 & 1 & 0.67 & $(-)$ & Equation 10 \\
\hline$T_{C A}$ & 70.4 & 54.3 & 45.2 & 76.4 & 63.0 & 55.2 & ${ }^{\circ} \mathrm{C}$ & Equation 5 \\
\hline$\dot{Q}_{C A}$ & 12.1 & 17.7 & 20.9 & 10.0 & 14.6 & 17.4 & MW & Equation 4 \\
\hline$\psi_{u} \max$ & 0.65 & 0.65 & 0.65 & 0.65 & 0.65 & 0.65 & $(-)$ & Figure 3 \\
\hline$\Omega_{\text {spec }, a}$ & 0.044 & 0.044 & 0.044 & 0.037 & 0.037 & 0.037 & $(-)$ & Figure 3 \\
\hline$\dot{W}_{\mathrm{NPO}}$ & 532 & 779 & 920 & 370 & 540 & 644 & kW(el) & Equation 12 \\
\hline$\dot{Q}_{1} \max$ & 7.9 & 11.5 & 13.6 & 6.5 & 9.5 & 11.3 & MW & Equation 2 \\
\hline
\end{tabular}

Assuming that the river water and brine has an equal specific heat capacity of $4,180 \mathrm{~J} / \mathrm{kg} \mathrm{K}$, as well as a density of $1,000 \mathrm{~kg} / \mathrm{m}^{3}$, the inverse apparent heat capacity of the heat source stream, $\alpha_{1}$, can be determined to $2.87 \mathrm{~K} / \mathrm{MW}$, according to Equation 9 .

As the flow of river water is unknown, three flow rates are investigated, 150, 300 and $450 \mathrm{~m}^{3} / \mathrm{h}$. This corresponds to $\alpha_{2}$ of 5.74, 2.87 and $1.91 \mathrm{~K} / \mathrm{MW}$ and $\alpha_{\text {rat }}$ of 2,1 and 0.67 respectively, as of Equation 10.

With the known data $\dot{Q}_{\mathrm{CA}}$ can be calculated according to Equation 4. Optimum utilization can be identified in Figure 3 and net power output, $\dot{W}_{\mathrm{NPO}}$ is calculated according to Equation 1. On top of that, we can calculate the maximum rate of heat transfer for the heat exchangers in the heat source, $\dot{Q}_{1}$, using Equation 2.

Figure 4 shows net power output and required heat transfer as a function of the flow rate of river water.

With this information, the engineer can create preliminary business cases by making estimations on yearly operating times, price of electric power, investment level, maintenance costs, interest rates, etcetera, in a conventional and simple manner.

Note that this information is of importance also in writing tenders for suppliers to make quotations on improving the quality of the quotes received considerably.

\section{Results and discussion}

This article shows that in low-temperature geothermal applications, the expected power output of any combination of finite heat source and finite heat sink can be estimated by two unique parameters. One, the ratio of entry temperatures in source and sink, defines the particular site or application. The second, utilization $\psi_{U}$, is the ratio of chosen, real heat transport from the heat source to the theoretical heat transport from the heat source if a reversible power cycle is assumed. By introducing the grouped term, application-specific work ratio or $\Omega_{\mathrm{spec}, a}\left(\psi_{U}\right)$, the calculation of expected power output is simplified to the degree of a graph shown in Figure 3. 


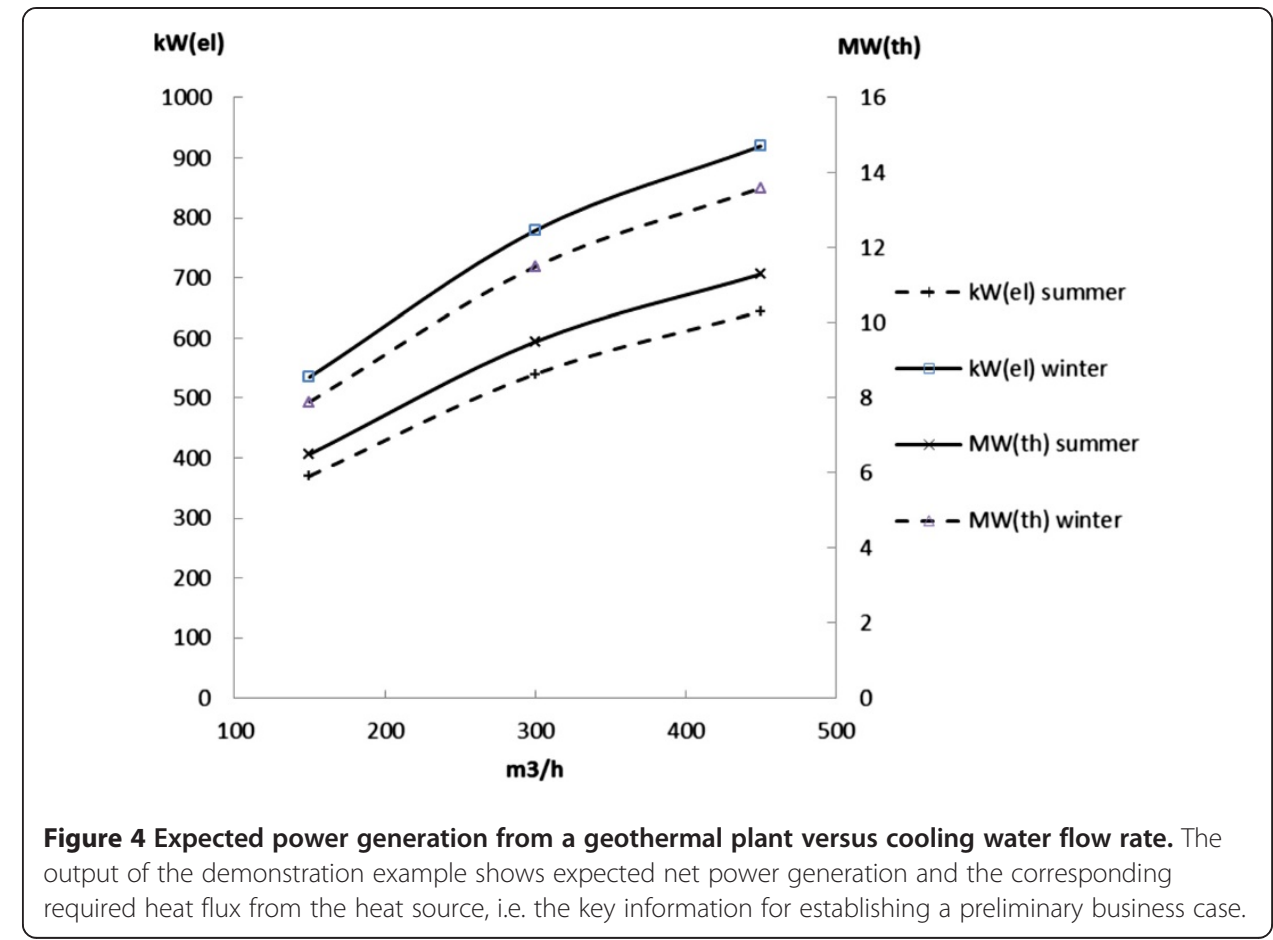

$\Omega_{\text {spec }, a}\left(\psi_{U}\right)$ is defined by Equation 13 and $\psi_{U}$ by Equation $2 . \Omega_{\text {spec, }, a}\left(\psi_{U}\right)$ indicates the ratio of expected net power out, at the particular utilization, to $\dot{Q}_{C A}$, the rate of heat transfer from the heat source at the utilization of 1 . Irreversibilities in real LTPCs are accounted for by the fraction of Carnot (FoC), as in Equation 7.

Figure 3 can be used to determine expected maximum power generation of any real LTPC as a function of the utilization ratio, see Equation 2. This is done by multiplying $\Omega_{\text {spec, } a}\left(\psi_{U}\right)$ with $\dot{Q}_{\mathrm{CA}}$ of Equation 4. This calculation is fully compatible to firstand second-law requirements while being simple to perform without expert thermodynamic knowledge. It is however limited to heat sources and heat sinks with constant apparent heat capacity. The calculation takes scientific correlation of practically achieved performances into account.

The simplified procedure to determine expected max power generation is as follows:

- Determine the maximum allowed rate of heat transfer from the heat source, assuming no losses, as of Equation 4 (requires source and sink temperature and flow stream information of the particular application).

- Determine the utilization providing the maximum power efficiency using Figure 3

- Calculate the expected power generation using Equation 12. Note that cases may occur when the optimal $\psi_{U}$ may not be technically or economically feasible. In such case, the closest available $\psi_{U}$ should be used and the corresponding $\Omega_{\mathrm{spec}, a}\left(\psi_{U}\right)$.

In the examples provided the reader may follow the procedure using real data.

One could argue that conventional exergy loss analysis or entropy generation analysis would offer better accuracy and more information. The authors' opinion is that, however, such detailed analysis is better suited in a later stage of a project, 
allowing detail optimization and creation of high-resolution performance validation points.

As is seen in Figure 3, the optimal level of $\psi_{U}$ is found in a narrow range. The explanation for this is likely to be found in two fundamental properties: the shape of the curves in Figure 1 in combination with the exponential form of Equation 7. A detailed analysis of this behaviour is beyond the scope of this article but would be of interest for a deeper understanding.

In more complex applications, when apparent heat capacities may vary with temperature, the same method can be applied but Figure 3 has to be recalculated according to Equation 1.

\section{Conclusions}

The conclusion of this investigation is that a simplified model is suitable to determine expected power output from any combination of finite heat source and finite heat sink using any type of real low-temperature power cycle.

Furthermore, this simplified model can be reduced to only require the input data of flow rate, heat capacity and entry temperature of the heat sink and heat source.

\section{Nomenclature}

LTPC: Abbreviation for Low Temperature driven Power Cycle

WHR: Abbreviation for Waste Heat Recovery

$\eta_{t h}$ : Thermal efficiency. Ratio of work to heat drawn from heat source (\%)

$\eta_{c, I l}$ : Integrated Local Carnot Efficiency (\%)

$\eta_{c, l}:$ Local Carnot Efficiency (\%)

$\eta_{C A}$ : Curzon-Ahlborn Efficiency (\%)

$\dot{Q}_{C A}:$ Rate of heat transfer from source at $\psi_{U}=1(\mathrm{~W})$

$\dot{Q}_{1}$ : Rate of heat transfer from source (W)

$T_{C A}$ : Common source and sink exit temperature with reversible process (K)

$\psi_{U}$ : Utilization of reversibly available heat transfer from source to sink by means of a reversible power cycle (-)

$T_{1}$ : Heat source entry temperature Brine temp $(\mathrm{K})$

$T_{2}$ : Heat sink entry temperature (K)

$\alpha_{1}$ : Inverse of apparent heat capacity of the source stream $(K / M W)$

$\alpha_{2}$ : Inverse of the apparent heat capacity of the sink stream $(K / M W)$

FoC: FractionOfCarnot Measure of irreversibility

$\dot{W}_{N P O}$ : Net Power Out (W)

$\Omega_{\text {spec }, \alpha}$ : Application specific work ratio. $\left(\dot{W}_{N P O}\right.$ to $\left.\dot{Q}_{C A}\right)(-)$

$T_{\text {rat }}$ : Ratio of heat sink and heat source entry temperatures (-)

$a_{\text {rat }}:$ Ratio of the inverse apparent heat capacities of the streams (-)

\section{Endnote}

${ }^{a}$ Notably, this term is identical to the Curzon-Ahlborn efficiency, or endo-reversible efficiency, $\eta_{\mathrm{CA}}$ (Curzon and Ahlborn 1975), when all of the potential heat transfer from source to sink is utilized. However, in real applications, all heat transfer cannot be utilized; therefore, integrated local Carnot efficiency is required for the analysis. A further 
development was made by (Wu and Kiang 1992) explaining the Curzon-Ahlborn power rate with simplified irreversibility and also using all of the potential heat transfer between a finite heat source and a finite heat sink.

Competing interests

The authors declared that they have no competing interests.

\section{Authors' contributions}

HÖ and PL have authored this article in companionship with shared efforts and resources. Both authors read and approved the final manuscript.

\section{Authors' information}

HÖ has a 23-year experience of introducing novel thermodynamic technology to existing industrial enterprises. Furthermore, HÖ is a PhD student at the Royal Institute of Technology, KTH, Stockholm. PL is professor in Applied Thermodynamics and Refrigeration, Department of Energy Technology at the Royal Institute of Technology, KTH, Stockholm. PL has 25 years of experience from analysis and development of thermodynamic cycles for energy conversion. PL is member of the Swedish Academy for engineering sciences and President for commission E2, Heat Pumps and Heat Recovery of the IIR, International Institute of Refrigeration.

\section{Author details}

'Department of Energy Technology, Royal Institute of Technology KTH Brinellv, 68 Stockholm 10044, Sweden.

${ }^{2}$ Department of Energy Technology, Royal Institute of Technology Brinellv, 68 Stockholm 10044, Sweden.

Received: 6 October 2013 Accepted: 10 March 2014

Published online: 26 April 2014

\section{References}

Aghahosseini S, Dincer I (2013) Comparative performance analysis of low-temperature Organic Rankine Cycle (ORC) using pure and zeotropic working fluids. J Appl Therm Eng 54(1):35-42

Bu X, Wang L, Li H (2013) Performance analysis and working fluid selection for geothermal energy-powered organic Rankine-vapor compression air conditioning. Geothermal Energy 1:2

Curzon FL, Ahlborn B (1975) Efficiency of a Carnot engine at maximum power output. Am J Phys 43:22-24

Fischer J (2011) Comparison of trilateral cycles and organic Rankine cycles. Energy 36:6208-6219

Heberle F, Preissinger M, Bruggemann D (2012) Zeotropic mixtures as working fluids in Organic Rankine Cycles for low-enthalpy geothermal resources. Renewable Energy 37:364-370

Ho T, Mao SS, Greif R (2012) Increased power production through enhancements to the Organic Flash Cycle (OFC). Energy 45:686-695

Öhman H, Lundqvist P (2012) Theory and method for analysis of low temperature driven power cycles. J Appl Therm Eng 37:44-50

Öhman H, Lundqvist P (2013) Comparison and analysis of performance using Low Temperature Power Cycles. J Appl Therm Eng 52:160-169

Schuster A, Karellas S, Kakaras E, Spliethoff H (2009) Energetic and economic investigation of Organic Rankine Cycle applications. J Appl Therm Eng 29:1809-1817

Vèles F, Chejne F, Antolin G, Quijano A (2012) Theoretical analysis of a transcritical power cycle for power generation from waste energy at low temperature heat source. J Enconman 60:188-195

Walraven D, Laenen B, D'haeseleer W (2013) Comparison of thermodynamic cycles for power production from low-temperature geothermal heat sources. J Enconman 66:220-233

Wu C, Kiang RL (1992) Finite-time thermodynamic analysis of a Carnot engine with internal irreversibility. Energy 17(12):1173-1178

doi:10.1186/s40517-014-0004-2

Cite this article as: Öhman and Lundqvist: Thermodynamic pre-determination of power generation potential in geothermal low-temperature applications. Geothermal Energy 2014 2:4.

\section{Submit your manuscript to a SpringerOpen ${ }^{\circ}$} journal and benefit from:

- Convenient online submission

- Rigorous peer review

- Immediate publication on acceptance

- Open access: articles freely available online

- High visibility within the field

- Retaining the copyright to your article

Submit your next manuscript at $\boldsymbol{\sim}$ springeropen.com 